\title{
THE EXPERIENCE OF A TERTIARY CENTER ON IGG4-RELATED DISEASE: TREATMENT, PROGNOSIS AND
} EPIDEMIOLOGIC FEATURES

Aline Kühl Torricelli (Hospital das Clínicas FMUSP São Paulo, Sao Paulo, SP, Brasil), Leandro Lara Prado (Hospital das Clínicas FMUSP São Paulo, São Paulo, SP, Brasil), Célio Roberto Gonçalves (Hospital das Clínicas FMUSP São Paulo, Sao Paulo, SP, Brasil)

\section{BACKGROUND}

- IgG4 related disease (IgG4-RD) is a "new" defined immune-mediated pathology identified in any organ or system with a variable clinical feature. Therapeutic approaches as well as prognostic factors have not yet been adequately established.

- $\quad$ The primary objective was to longitudinally evaluate clinical response, imaging and laboratory of IgG4-RD patients after glucocorticoid and immunosuppressor treatments.

- Secondarily, to assess which factors impact treatment refractoriness and compare our epidemiologic data with literature.

\section{MATERIALS AND METHODS}

- $\quad$ Single center retrospective study at a rheumatology outpatient clinic from a tertiary hospital.

- $\quad$ The clinical, imaging and laboratorial data were obtained through a systematic chart review from an electronic database.

- $\quad$ Subjects fulfilled the diagnostic criteria for IgG4-RD and were followed up from diagnosis up to 12 months.

- Data were collected at diagnosis, after 6-month and 12-month treatment.

- $\quad$ T-student test, McNemar's test, ANOVA, Fisher's exact test and Mann-Whitney were used for comparisons. Statistical relevance was considered when $\mathrm{p}<0.05$.

\section{RESULTS}

- $\quad$ Seventeen patients were selected for this study. According to diagnostic criteria: one patient had definite IgG4-RD, five possible and eleven probable IgG4-RD.

- $\quad$ Regarding epidemiologic features, our cohort were predominantly male (10/58.9\%), with a median age of 51.9 years $( \pm 14.5)$.

- As for IgG4-RD clinical manifestations, mostly had localized disease (14/82.3\%) and the distribution of impairment were, as follows: hypophysitis (5/29.4\%) retroorbital pseudotumor (4/23.5\%), retroperitoneal fibrosis $(4 / 23.5 \%)$, pancreatitis (3/17.6\%), dacryoadenitis (1/5.8\%), oropharynx pseudotumor (1/5.8\%), skin (1/5.8\%), Mikulicz (1/5.8\%), mastoiditis (1/5.8\%).

- Longitudinal evaluation after treatment with glucocorticoids and immunosuppressors showed: reduction of corticosteroids dosage at 6 months $(p<0.001)$ and at 12 months $(p<0.001)$ when compared to the initial dose $(1 \mathrm{mg} / \mathrm{kg})$; image improvement or stability in 6 months ( $p=0.04$ ) as well as in 12 months 
$(p=0.013)$; , decrease CRP levels to normal baseline in 6 months ( $p=0.002)$, clinical improvement (defined by improvement of signs and symptons) in 6 months ( $p=0.004)$.

- $\quad$ IgG4-RD patients with pancreatitis showed a possible predictive response factor $(p=0.051)$, with lack of clinical improvement in 6 months .

\section{CONCLUSION}

- $\quad$ IgG4-RD treatment with glucocorticoids plus immunosuppressors had a favorable outcome at 6 and 12-month follow-up regarding imaging, clinical response, glucocorticoids reduction and CRP decrease.

- $\quad$ IgG4-RD pancreatitis seems to be a refractoriness clinical factor. The reduced number of patients may be a limitatioon, therefore, more studies should be done to confirm this finding.

- $\quad$ Our cohort was younger than reported in literature (between the sixth and seventh decades of life) but with similar male predominance. Localized disease, hypophysitis, retroorbital pseudotumor and retroperitoneal fibrosis were more frequent in our casuistic. 